\title{
Dynamic Monitoring Method of Coconut Red Ring Disease based on Apriori Algorithm
}

\author{
Xiao Heng a and Gautam Srivastava ${ }^{\text {b,* }}$ \\ ${ }^{a}$ School of Information and Intelligent Engineering Sanya University, Sanya, 572000, China \\ ${ }^{b}$ Department of Mathematics and Computer Science, Brandon University, Brandon, R7A 6A9, Canada
}

\begin{abstract}
In order to improve the dynamic monitoring and feature recognition ability of coconut red ring disease, the image visual feature recognition method is used to detect the disease, and the method of coconut red ring disease feature recognition based on the Apriori algorithm is proposed. A two-dimensional dynamic hyperspectral image acquisition model of coconut red ring disease is constructed. The dynamic hyperspectral images of the disease are detected by block fusion, while the features are detected according to its texture distribution. The visual fractal features are extracted, the surface texture registration and block regional feature matching method are used to calibrate the feature points, and the feature decomposition of dynamic hyperspectral images are carried out by multi-scale wavelet decomposition. The simulation results show that the accuracy of the method for the identification and dynamic monitoring of coconut red ring disease is high, and the false detection rate is low, which improves the ability of prevention and recognition of coconut red ring disease.
\end{abstract}

Keywords: apriori algorithm; machine vision; image; coconut; red ring disease; feature recognition; dynamic monitoring

(Submitted on October 28, 2019; Revised on November 30, 2019; Accepted on December 16, 2019)

(C) 2019 Totem Publisher, Inc. All rights reserved.

\section{Introduction}

With the development of image processing technology and machine vision information processing technology, it is possible to use machine vision analysis method to control coconut red ring disease in plants. Coconut red ring disease has strong concealment and greatly influences coconut yield [1-2]. The conventional detection and control technology of coconut red ring disease is not effective in controlling the disease. The research on the characteristics recognition method of coconut red ring disease is of great significance to promote its prevention and control [3].

In reference [4], a multifractal-based dynamic hyperspectral image feature extraction technique for coconut red ring disease was proposed. The sub-regional feature matching method was used to detect the block fusion of two-dimensional coconut red ring disease dynamic hyperspectral images, and the green leaf texture fractal was used to realize the feature extraction. However, the anti-interference of this method was not good. The dynamic fusion method was used to detect the visual images of coconut red ring disease. In reference [5], a visual image edge contour detection method of coconut red ring disease based on multiple texture fusion was proposed. The fuzzy correlation feature detection method was used to realize medical image detection, but the computational complexity of the method was high and the feature resolution was not good. The visual image dynamic monitoring method of coconut red ring disease based on two-dimensional color space block matching was proposed in reference [6]. The visual image acquisition and feature projection processing of coconut red ring disease were carried out. Additionally, the binary fitting of coconut red ring disease visual images was carried out to realize the dynamic monitoring of the images. However, the fuzzy degree of this method was large, and the self-adaptability was not optimal.

In order to solve the above problems, a feature recognition method of coconut red ring disease based on the Apriori algorithm is proposed in this paper.

\footnotetext{
* Corresponding author.

E-mail address: srivastavag@brandonu.ca
} 


\section{Dynamic Hyperspectral Image Block Fusion of Coconut Red Ring Disease}

On the basis of constructing a two-dimensional dynamic hyperspectral image acquisition model of coconut red ring disease, the dynamic hyperspectral images of coconut red ring disease are detected by block fusion. The distribution matrix of pixel sequence of $3 \mathrm{D}$ coconut red ring disease visual images is described by using the green leaf texture regional detection method.

$$
D=\left[\begin{array}{cc}
I_{x}^{2} & I_{x} I_{y} \\
I_{x} I_{y} & I_{y}^{2}
\end{array}\right]
$$

By using the cost constraint analysis method, the sampling point density of the visual images of coconut red ring disease is obtained as follows:

$$
E\left(\mathbf{T}_{n}\right)=\frac{1}{N} \sum_{i=0}^{N-1}\left\|p_{i}-\mathbf{T}_{n}\left(g_{i}^{\prime}\right)\right\|^{2}+\tau \cdot \Phi\left(\mathbf{T}_{n}\right)
$$

By using the adaptive weighted control method, the inverse weighted $f\left(g_{i}\right)$ of the visual images of coconut red ring disease is obtained as follows:

$$
f\left(g_{i}\right)=c_{1} \tilde{\lambda}_{i} \sum_{j=0}^{N_{n p}} \frac{\rho_{j} \vec{v}_{i j}}{\left|\vec{v}_{i j}\right|^{\sigma_{1}}+\varepsilon} / \sum_{j=0}^{N_{n p}} \frac{\rho_{j}}{\left|\vec{v}_{i j}\right|^{\sigma_{1}}+\varepsilon}
$$

The interpolation transformation of coconut red ring disease visual images is carried out in the Taubin smoothing region, and the edge outline feature decomposition is obtained by using the model vertex matching method [7]. The expression of edge outline feature decomposition of coconut red ring disease visual images is obtained as follows:

$$
\begin{aligned}
& f\left(\mathbf{G}_{n}\right)=a_{1}+a_{2} x+a_{3} y+a_{4} z+\sum_{i=0}^{n} \gamma_{i} U\left(g_{i}^{\prime}, p_{i}\right) \\
& g\left(\mathbf{G}_{n}\right)=b_{1}+b_{2} x+b_{3} y+b_{4} z+\sum_{i=0}^{n} \theta_{i} U\left(g_{i}^{\prime}, p_{i}\right) \\
& h\left(\mathbf{G}_{n}\right)=c_{1}+c_{2} x+c_{3} y+c_{4} z+\sum_{i=0}^{n} \omega_{i} U\left(g_{i}^{\prime}, p_{i}\right)
\end{aligned}
$$

Wherein

$$
\Phi\left(\mathbf{T}_{n}\right)=\boldsymbol{\gamma}^{T} \mathbf{H} \gamma+\boldsymbol{\theta}^{T} \mathbf{H} \boldsymbol{\theta}+\boldsymbol{\omega}^{T} \mathbf{H} \boldsymbol{\omega}
$$

According to the above model, the visual image outline reconstruction of coconut red ring disease is carried out, and the pixel reconstruction of the edge outline region is carried out in the gray pixel region. The visual feature fusion of coconut red ring disease is realized, and the feature recognition is realized according to the block detection results [8].

\section{Optimization of Feature Recognition of Coconut Red Ring Disease}

\subsection{Calibration of the Characteristics of Coconut Red Ring Disease}

Based on the above construction of the two-dimensional coconut red ring disease dynamic hyperspectral image acquisition model and the collection of coconut red ring disease dynamic hyperspectral image block fusion detection, the coconut red ring disease feature recognition method based on the Apriori algorithm is proposed in this paper [9]. The wavelet noise reduction method is used to separate the original $3 \mathrm{D}$ coconut red ring vision images. The linear equations of image wavelet are as follows:

$$
\left(\begin{array}{lll}
\mathbf{x} & \mathbf{y} & \mathbf{z} \\
\mathbf{0} & \mathbf{0} & \mathbf{0}
\end{array}\right)=\left(\begin{array}{cc}
(\mathbf{H}+\tau \cdot \mathbf{I}) & \mathbf{P} \\
\mathbf{P}^{T} & \mathbf{O}
\end{array}\right)\left(\begin{array}{ccc}
\gamma & \boldsymbol{\theta} & \boldsymbol{\omega} \\
\mathbf{a} & \mathbf{b} & \mathbf{c}
\end{array}\right)
$$


On the reconstruction surface of the images, the Harris corner detection method is used to smooth the visual images of coconut red ring disease. The smoothing function is expressed as follows:

$$
D(x, y, \sigma)=(G(x, y, k \sigma)-G(x, y, \sigma)) * I(x, y)=L(x, y, k \sigma)-L(x, y, \sigma)
$$

Wherein

$$
L(x, y, \sigma)=G(x, y, \sigma) \otimes I(x, y)
$$

$I(x, y)$ represents the grayscale value of the visual image of coconut red ring disease at $(x, y), L(x, y, \sigma)$ represents the Taubin smoothing operator, and the block matching characteristic coefficient of the 3D coconut red ring disease image is $G(x, y, \sigma)$. The calculation formula is as follows:

$$
G(x, y, \sigma)=\frac{1}{2 \pi \sigma^{2}} e^{-\frac{\left(x^{2}+y^{2}\right)}{2 \sigma^{2}}}
$$

Judging the fold information of coconut red ring disease visual images, according to the Euclidean distance $E(d(x, y))$ between folds, the image noise level decomposition is carried out, and the decomposition coefficient is obtained as follows:

$$
F_{d}-\frac{d}{d x} F_{d_{x}}-\frac{d}{d y} F_{d_{y}}=0
$$

In the formula, $F_{d}$ represents the edge scale of the visual image of coconut red ring disease, and the noise distance of the grid surface of the image is $d(x, y)$. The noise points of the coconut visual image, $N_{l}$, are described as follows:

$$
N_{l}= \begin{cases}1, & l=0, L \\ {\left[2 \pi \cdot \frac{D}{2} \cdot \sin \eta / l_{\text {triangle }}\right],} & l=1, \cdots, L-1\end{cases}
$$

According to the above analysis, the noise separation model is used to smooth the visual images of coconut red ring disease. The mathematical expression of visual image output of coconut red ring disease after smooth filtering is as follows:

$$
\begin{gathered}
\mathbf{G}_{\text {new }}=(1+\mu T)(1+\lambda T) \mathbf{G}_{\text {old }} \\
T\left(g_{i}\right)=\frac{1}{\sum_{k} \omega_{k}} \cdot \sum_{k} \omega_{k}\left(g_{k}-g_{i}\right)
\end{gathered}
$$

Where $\mathbf{G}_{\text {new }}$ and $\mathbf{G}_{\text {old }}$ represent the low frequency component and high frequency component of coconut red ring disease visual images, respectively. The characteristics of coconut red ring disease are sorted, and the visual reconstruction and recognition of the regional distribution of coconut red ring disease are carried out [10-11].

\subsection{Detection of Characteristics of Coconut Red Ring Disease}

The multi-scale wavelet feature decomposition method is used to decompose the dynamic hyperspectral images of coconut red ring disease [12-13]. The wavelet function of the dynamic hyperspectral images is expressed as follows:

$$
R(x, y)=x^{2}+y^{2}+d x+e y+f
$$

In the maximum search region, the gray scale invariant moment of the dynamic hyperspectral images of coconut red ring disease is obtained as 


$$
\left\{\begin{array}{l}
\frac{\partial R}{\partial x}=2 x+d=0 \\
\frac{\partial R}{\partial y}=2 y+e=0
\end{array}\right.
$$

According to the error distribution, the block region reconstruction model of coconut red ring disease dynamic hyperspectral images is constructed, and the characteristic resolution intensity is obtained.

$$
\begin{aligned}
I_{G S M} & =I\left(C^{N} ; D^{N} \mid s^{N}\right) \\
& =\sum_{i=1}^{N} I\left(C_{i} ; D_{i} \mid s_{i}\right) \\
& =\sum_{i=1}^{N}\left(h\left(D_{i} \mid s_{i}\right)-h\left(D_{i} \mid C_{i}, s_{i}\right)\right) \\
& =\sum_{i=1}^{N}\left(h\left(g_{i} C_{i}+V_{i} \mid s_{i}\right)-h\left(V_{i}\right)\right)
\end{aligned}
$$

The visual feature recognition and reconstruction of coconut red ring disease are realized, and the feature recognition of coconut red ring disease is realized according to the reconstruction results.

\section{Fine Particle Size Feature Extraction of Red Ring Lesion Cells}

The edge outline feature extraction method is used to extract the fine-grain features of the red ring lesion cells in the visual images of coconut red ring disease [14-15]. The matching points on the visual image grid model are calculated. The multi-component transfer function expression of the visual images of coconut red ring disease is designed as follows:

$$
g_{i}^{*}=\left\{\begin{array}{c}
R s_{j}, z \leq i \leq x-y \\
g_{i}, \quad \text { otherwise }
\end{array}\right.
$$

In this formula, the edge pixel point set of coconut red ring disease visual images is represented, and a canonical constant is represented, which represents the ambiguity coefficient of coconut red ring disease visual images and the two dimensional outline feature matching coefficient of the images [16-17]. The RGB decomposition of coconut red ring disease visual images is carried out by using multi-component pixel spatial feature recombination and quantitative decomposition method. In the position RGB feature component, the gray pixel level of the visual images of coconut red ring disease is obtained, and the cell distribution eigenvalues are calculated. According to the template shape, the visual images are matched by blocks [18-19]. The pixel set intensity is obtained.

$$
n_{p q}=\frac{\mu_{p q}}{\left(\mu_{00}\right)^{\gamma}}
$$

Where $\mu_{p q}$ represents the central moment of the visual images of coconut red ring disease, $\mu_{00}$ represents the zero order moment of fine granularity feature extraction of red ring lesion cells in the visual images, and $\gamma$ represents the order. The edge outline feature of coconut red ring disease visual images is detected by the color template feature matching method. At the center pixel point SF of the distribution of coconut red ring disease, the center moment of dynamic monitoring of visual images is $v_{i}$. The average distance of the pixels of the grid model is calculated by taking the pixel point PE1 for the two-dimensional profile feature distribution point $\left(x_{i+1}, y_{i+1}\right)$. The edge profile feature distribution function $\left(x_{i}+\frac{1}{2}, y_{i+1}\right)$ of the coconut red ring disease visual images is expressed as follows:

$$
F_{d} \delta_{l}^{2}-\frac{d}{d x} F_{d_{x}}-\frac{d}{d y} \beta F_{d_{y}}=0
$$


Where $F_{d}$ represents the edge scale of tone mapping, $\delta_{l}^{2}$ is the local variance of the coconut red ring disease visual image, $\delta_{\eta}^{2}$ is the response function, and $\beta=\max \left[\frac{\delta_{l}^{2}-\delta_{\eta}^{2}}{\delta_{l}^{2}}, 0\right]$ carries out two-dimensional reconstruction of coconut red ring disease visual images in the high dynamic range. The feature component of color space satisfies the pixel gray value of $C \in S$, with image synthesis and hue mapping, and the pixel gray value at the $m$ frame $(x, y)$ of the coconut red ring disease visual image $F_{m}(x, y)$ is obtained. The fine particle size feature extraction of red ring lesion cells is realized.

\section{Apriori Algorithm and Dynamic Monitoring Optimization of Coconut Red Ring Disease}

The ultra-fine particle size feature of coconut red ring disease visual images is extracted, and the adaptive optimization of coconut red ring disease visual image dynamic monitoring is carried out by using the Apriori algorithm. The depth learning process of coconut red ring disease visual images is described as follows:

$$
\operatorname{Dif}\left(C_{1}, C_{2}\right)=\min _{\left.v_{i} \in C_{1}, v_{j} \in C_{2}, v_{i}, v_{j}\right) \in E} n w\left(v_{i}, v_{j}\right)
$$

In the above formula, $n=1,2, \cdots, T$ represents the number of iterative steps for the dynamic monitoring of coconut red ring disease visual images. The gray pixel subset $C \subseteq V$ uses the weighted average method to detect the color and texture of the images. The statistical feature distribution function of coconut red ring disease visual images is described by $I(i, j)$, and the $I_{(k)}(i, j)$ representation is as follows:

$$
I(i, j)=\sum_{k=1}^{P} I_{(k)}(i, j) \times 2^{k-1}
$$

Where $k$ represents the order of the spatial position of the visual images of coconut red ring disease. According to the segmentation of the visual images into $2 \times 2$ sub-blocks of $M \times N$, the wavelet multi-scale decomposition method is used to reconstruct the visual images [20]. The low dynamic range detection sequence of coconut red ring disease visual images is output as follows:

$$
G_{m, n}=\left(\begin{array}{ll}
p_{(m, n)}(1,1) & p_{(m, n)}(1,2) \\
p_{(m, n)}(2,1) & p_{(m, n)}(2,2)
\end{array}\right), m=1,2, \cdots, M, n=1,2, \cdots, N
$$

Wherein

$$
p_{(i, j)}(u, v)=I_{(k) g}[2(i-1)+u, 2(j-1)+v], u \in\{1,2\}, v \in\{1,2\}
$$

Where $u$ is the gray pixel value of the coconut red ring disease visual image $p(i, j)$ in the joint affine invariant region, and $(i, j)$ is the coordinate value of the corresponding pixel. Through the design of the above algorithm, the edge profile of coconut red ring disease visual images is detected, and the Apriori algorithm is used to optimize the visual image. The optimization coefficient in the process of deep learning is obtained.

$$
h=\theta / \pi, \quad v=\lambda_{1}+\lambda_{2}, \quad s=1-\frac{\lambda_{2}}{\lambda_{1}}
$$

According to the optimization design of the above algorithm, the dynamic monitoring algorithm of coconut red ring disease visual images is improved.

\section{Simulation Experiment and Result Analysis}

In order to test the application performance of this method in realizing the feature recognition of coconut red ring disease, the simulation software platform of the experiment is $\mathrm{C}++$. in the experiment, the optical sensor LBP Sensors is used to program the image information processing of coconut red ring disease with Visual $\mathrm{C}++6.0 \mathrm{MFC}$, and the feature 
recognition of coconut red ring disease is carried out under machine vision. The initial sampling pixel intensity of the internal characteristics of the coconut red ring disease area is 120 days, the image shift value is 1.24 , the energy intensity is $1.8 \mathrm{KJ}$ in the coconut red ring disease area, the resolution of image is $430 \times 210$, the initial evaluation parameter of disease pest detection is 3.5 , and the signal-noise ratio is $-30 \mathrm{~dB}$.

According to the above parameters, the feature recognition and extraction of coconut red ring disease are studied, and the two-dimensional dynamic hyperspectral image acquisition model of coconut red ring disease is constructed. The collected dynamic hyperspectral images of coconut red ring disease are detected by block fusion, and the image acquisition results are shown in Figure 1.

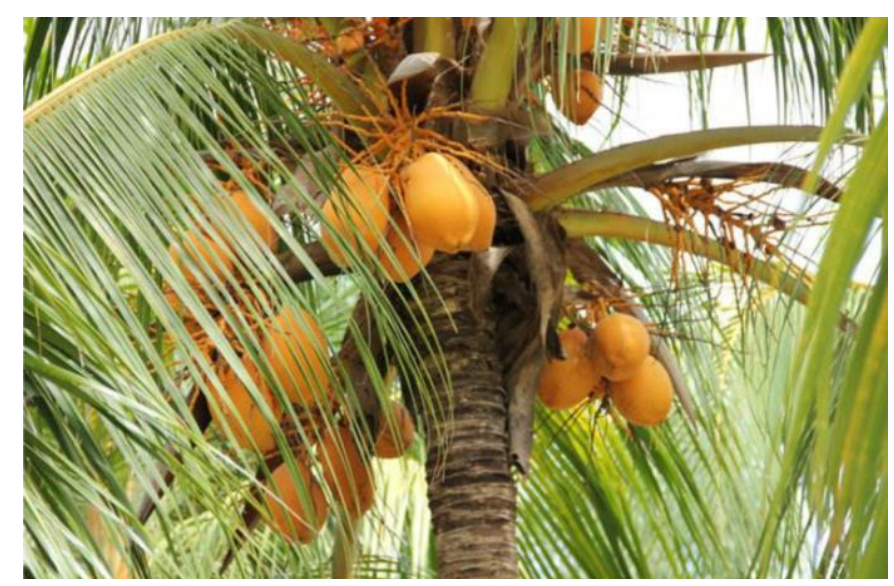

Figure 1. Dynamic hyperspectral image acquisition results of coconut red ring disease

The visual fractal feature quantity of Coconut Erythrocyclis is extracted from the images of the set of Figure 1, and surface texture registration and the block region feature matching method are used to calibrate the characteristic points of Coconucomatosis. The image fusion results are shown in Figure 2.

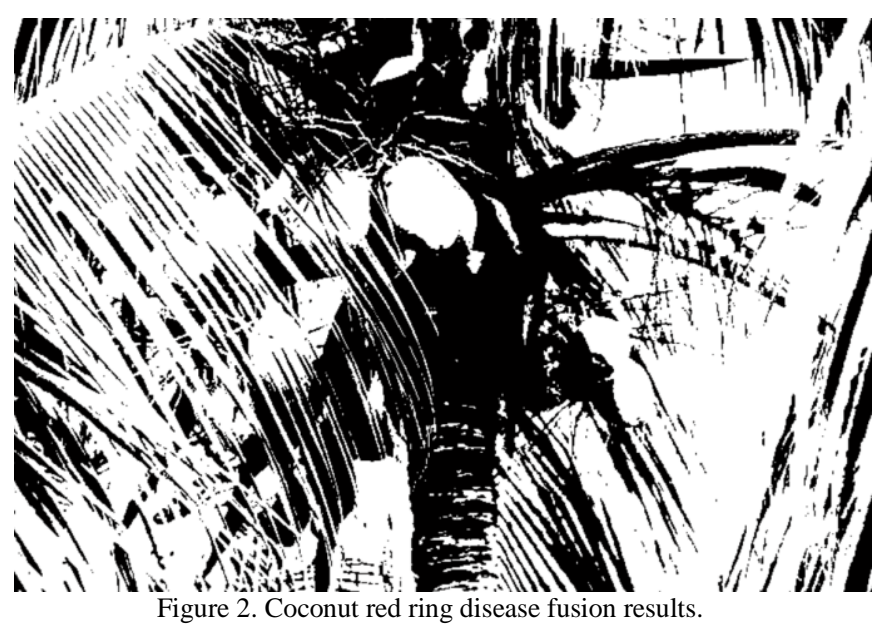

Figure 2 shows that this method can effectively reconstruct the visual features of coconut red ring disease. According to the reconstruction results, the feature extraction and recognition of coconut red ring disease can be realized, and the feature extraction results are shown in Figure 3.

In Figure 3, the characteristics of coconut red ring disease under each image shift parameter are effectively identified, which improves the feature detection and recognition ability of coconut red ring disease. Furthermore, the number of iterations for the visual image of coconut red ring disease is 120 , the number of samples sampled is 214 , the initial learning rate of visual image detection of coconut red ring disease is 0.12 , and the attenuation weight coefficient is 0.001 . Taking a laser image of coconut red ring disease as an example, the visual image is detected, and the original image is obtained as shown in Figure 4. 


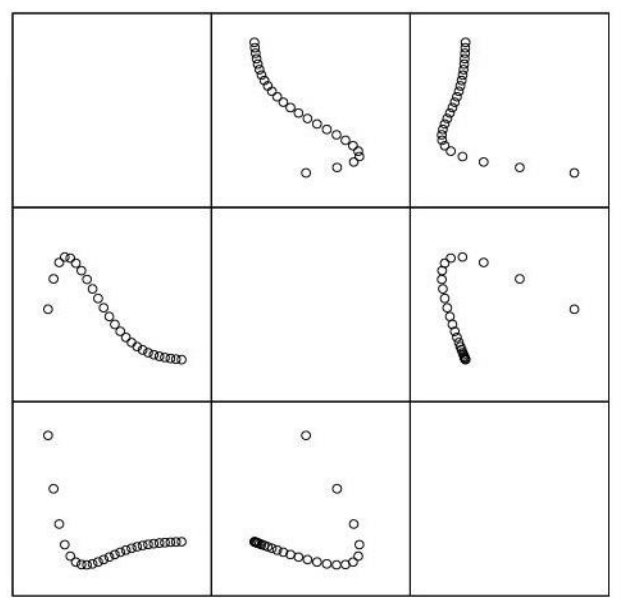

Figure 3. Recognition results of coconut red ring disease

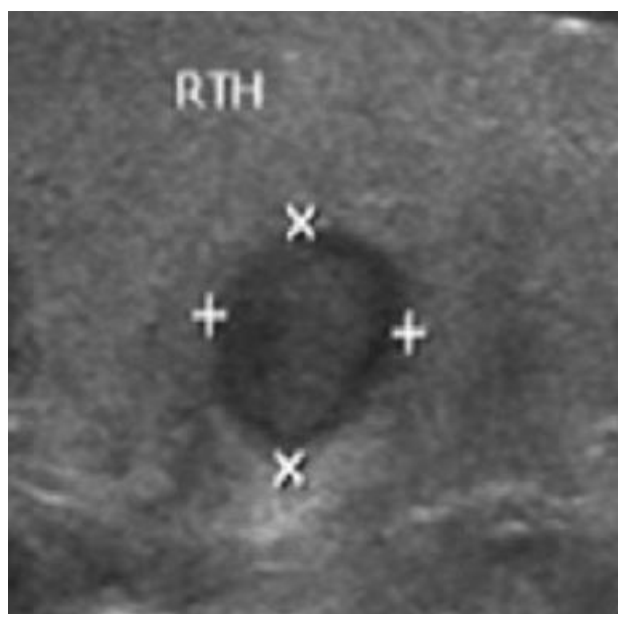

Figure 4. Original image

Taking the image of Figure 4 as the research sample, the 548 coconut red ring disease image is selected as the test sample set, the coconut red ring disease visual image is screened, and the three-dimensional surface structure reconstruction model of coconut red ring disease visual image is constructed. Combined with the ambiguity feature decomposition method, the multi-scale color structure feature decomposition and detection of coconut red ring disease visual images are carried out, and the fine-grain feature fusion results are obtained as shown in Figure 5.

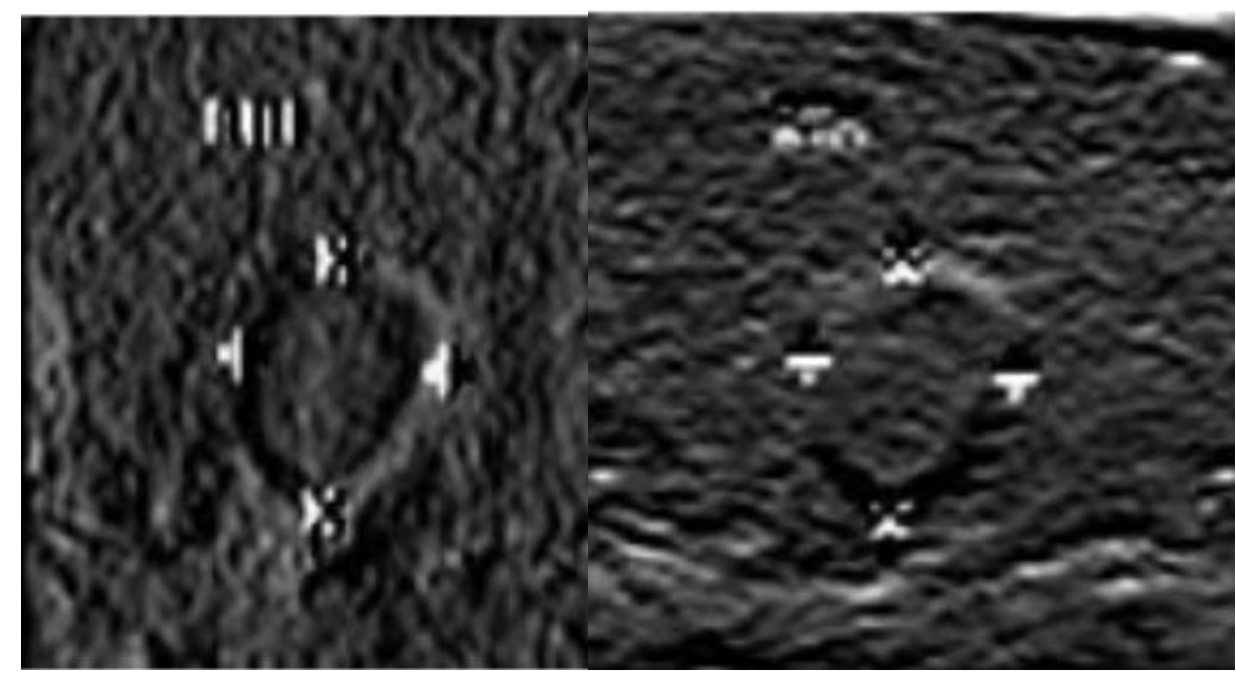

Figure 5. Fine granularity feature fusion results of visual images of coconut red ring disease 
Figure 5 shows that the detection and information fusion of fine particle size of red ring lesion cells in coconut red ring disease visual images can be effectively realized by using this method. On this basis, the dynamic monitoring of coconut red ring disease visual images is realized, and the detection output is shown in Figure 6.

The analysis of Figure 6 shows that this method has high adaptive convergence, strong feature resolution, and good detection and resolution of coconut red ring disease cells when monitoring the visual images of coconut red ring disease. The accuracy of different methods for visual image detection of coconut red ring disease is tested, and the comparative results are shown in Table 1. The analysis shows that the accuracy of this method is higher than that of coconut red ring disease visual image detection.

In order to compare the performance, the characteristics of coconut red ring disease are identified by this method and the traditional method under different image shift distribution. The false detection rate of coconut red ring disease is tested, and the comparison results are shown in Figure 7.
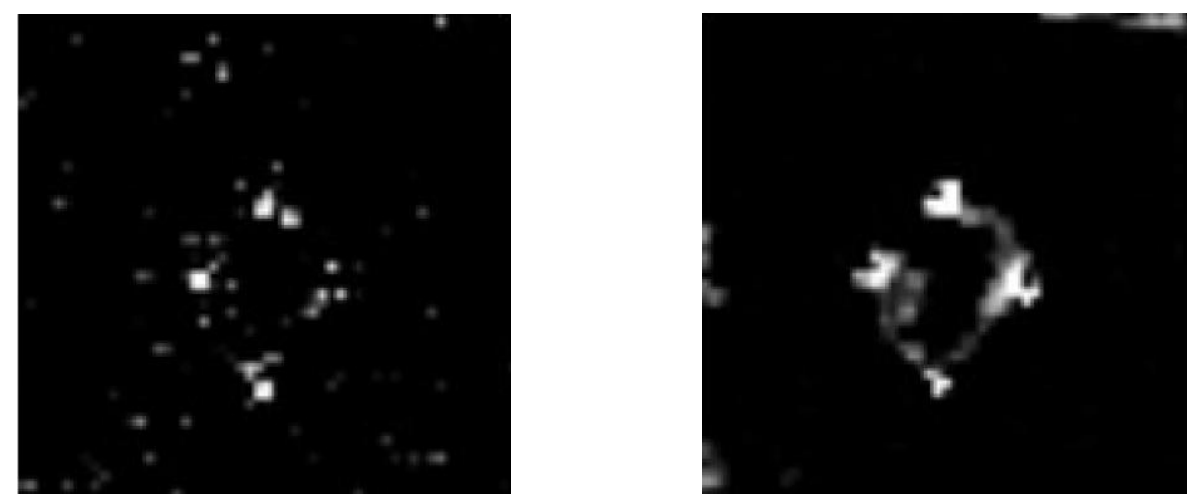

Figure 6. Dynamic monitoring output of the effect of coconut red ring disease

\begin{tabular}{cccc}
\multicolumn{3}{c}{ Table 1. Comparison of detection performance } & Reference [5] \\
\hline Sample number & Proposed method & Reference [4] & 0.835 \\
200 & 0.923 & 0.866 & 0.868 \\
300 & 0.945 & 0.890 & 0.911 \\
\hline
\end{tabular}

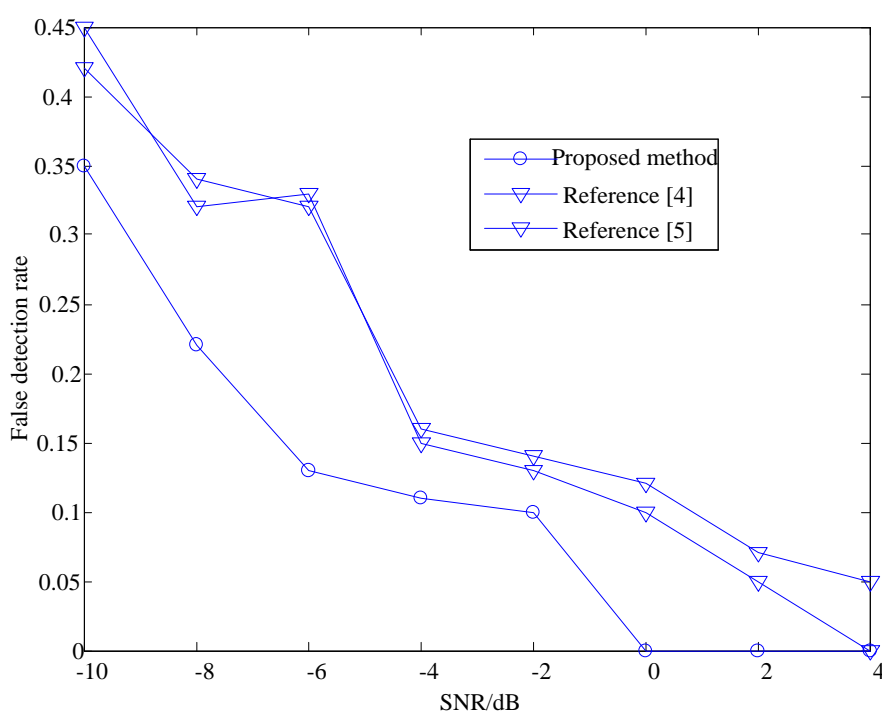

Figure 7. Comparison of false detection rates

Figure 7 shows that the false detection rate of coconut red ring disease feature detection and recognition by this method is low. The time cost of coconut red ring disease feature recognition by different methods is tested. The comparison results, which are shown in Table 2, indicate that the time cost of coconut red ring disease feature recognition by this method is short. 
Table 2. Time cost comparison (ms)

\begin{tabular}{cccc}
\hline Sample number & Proposed method & Reference [4] & Reference [5] \\
\hline 100 & 1.76 & 2.43 & 5.76 \\
200 & 1.98 & 4.65 & 7.83 \\
300 & 2.32 & 8.54 & 10.32 \\
\hline
\end{tabular}

\section{Conclusions}

In this paper, the image information processing model of coconut red ring disease is constructed. The detection and recognition of coconut red ring disease is carried out by using the dynamic monitoring image processing method, which can improve the regional prevention and control ability of the disease and a feature recognition method on the Apriori algorithm is proposed. The gray histogram feature decomposition method is used to reconstruct the visual features of coconut red ring disease. According to the texture distribution of the disease, the feature detection and texture matching are carried out. The statistical shape model of dynamic hyperspectral images of coconut red ring disease is established, and the feature recognition of coconut red ring disease under machine vision is realized according to the difference of color gradient change. The results show that the method has high accuracy, a low false detection rate, and good real-time performance in feature recognition of coconut red ring disease.

\section{Acknowledgments}

This paper is supported by the Natural Science Foundation of Hainan Province (No. 619QN243, 617182) and the Beichuang TA Project Fund (No. 2018A 01003).

\section{References}

1. K. Murphy, B. Van Ginneken, and A. M.Schilham, "A Large-Scale Evaluation of Automatic Pulmonary Nodule Detection in Chest CT using Local Image Features and k-Nearest-Neighbour Classification,” Medical Image Analysis, Vol. 13, No. 5, pp. 757-770, 2009

2. T. Lopez, E. Florina, and F. Pennazio, "Large Scale Validation of the M5L Lung CAD on Heterogeneous CT Datasets," Medical Physics, Vol. 42, No. 4, pp. 1477-1489, 2015

3. Y. Tu, Y. Lin, J. Wang, and J. U. Kim, "Semi-Supervised Learning with Generative Adversarial Networks on Digital Signal Modulation Classification," CMC-Computers Materials and Continua, Vol. 55, No. 2, pp. 243-254, 2018

4. Q. Dou, H. Chen, and L. Q. Yu, "Multi-Level Contextual 3D CNNs for False Positive Reduction in Pulmonary Nodule Detection," IEEE Transactions on Biomedical Engineering, Vol. 64, No. 7, pp. 1558-1567, 2017

5. H. T. Xie, D. B. Yang, and N. N. Sun, "Automated Pulmonary Nodule Detection in CT Images using Deep Convolutional Neural Networks," Pattern Recognition, Vol. 1, No. 85, pp. 109-119, 2019

6. W. Li, P. Cao, and D. Z. Zhao, "Pulmonary Nodule Classification with Deep Convolutional Neural Networks on Computed Tomography Images," Computational and Mathematical Methods in Medicine, Vol. 1, No. 62, pp. 80-85, 2016

7. J. Ding, A. X. Li, and Z. Q. Hu, "Accurate Pulmonary Nodule Detection in Computed Tomography Images using Deep Convolutional Neural Networks," in Proceedings of the 2017 International Conference on Medical Computing and Computer-Assisted Intervention, Vol. 1, No. 1, pp. 559-567, Berlin, Springer, 2017

8. K. Kamnisas, C. Ledig, and V. F. Newcombe, "Efficient Multi-Scale 3D CNN with Fully Connected CRF for Accurate Brain Lesion Segmentation,” Medical Image Analysis, Vol. 1, No. 36, pp. 61-78, 2016

9. Q. Dou, H. Chen, and L. Q. Yu, "Automatic Detection of Cerebral Microbleeds from MR Images via 3D Convolutional Neural Networks," IEEE Transactions on Medical Imaging, Vol. 35, No. 5, pp. 1182-1195, 2016

10. H. Chen, Q. Dou, and L. Q. Yu, "VoxResNet: Deep Voxelwise Residual Networks for Brain Segmentation from 3D MR Images," NeuroImage, Vol. 1, No. 170, pp. 446-455, 2018

11. O. Ronneberger, P. Fischer, and T. Brox, "U-Net: Convolutional Networks for Biomedical Image Segmentation," in Proceedings of the 2015 International Conference on Medical Computing and Computer-Assisted Intervention, Vol. 1, No. 1, pp. 234-241, Berlin, Springer, 2015

12. G. Yang and S. Liu, "Distributed Cooperative Algorithm for k-M Set with Negative Integer k by Fractal Symmetrical Property," International Journal of Distributed Sensor Networks, Vol. 4, pp. 1-7, 2014

13. C. H. Sudre, W. Q. Li, and T. Vercauteren, "Generalised Dice Overlap as a Deep Learning Loss Function for Highly Unbalanced Segmentations," in Proceedings of the 2017 International Conference on Deep Learning in Medical Image Analysis and Multimodal Learning for Clinical Decision Support, Vol. 1, No. 1, pp. 240-248, Berlin, Springer, 2017

14. G. Huang, Z. Liu, and V. D. M. Laurens, "Densely Connected Convolutional Networks," in Proceedings of the 2017 IEEE Conference on Computer Vision and Pattern Recognition, Ishington, DC: IEEE Computer Society, Vol. 1, No. 1, pp. 2261-2269, 2017

15. W. Huixian, J. Huijia, W. Jiaolong, and J. Wanshou, "Optimization Approach for Multi-Scale Segmentation of Remotely Sensed Imagery under k-Means Clustering Guidance,” Acta Geodaetica et Cartographica Sinica, Vol. 44, No. 5, pp. 526-532, 2015 
16. S. Liu, Z. J. Li, and X. C. Cheng, "Introduction of Recent Advanced Hybrid Information Processing," Mobile Networks and Applications, Vol. 23, No. 4, pp. 673-676, 2018

17. Y. Lin, C. Wang, J. X. Wang, and Z. Dou, "A Novel Dynamic Spectrum Access Framework based on Reinforcement Learning for Cognitive Radio Sensor Networks,” Sensors, Vol. 16, No. 10, pp. 1675, 2016

18. S. Liu, "Introduction of Key Problems in Long-Distance Learning and Training," Mobile Networks and Applications, Vol. 24, No. 1, pp. 1-4, 2019

19. Y. Lin, Y. Li, X. Yin, and Z. Dou, "Multisensor Fault Diagnosis Modeling based on the Evidence Theory," IEEE Transactions on Reliability, Vol. 67, No. 2, pp. 513-521, 2018

20. Z. W. Zhou, R. Mahfuzur, M. Siddiquee, and J. M. Liang, "UNet++: A Nested U-Net Architecture for Medical Image Segmentation," in Proceedings of the 2018 International Conference on Deep Learning in Medical Image Analysis and Multimodal Learning for Clinical Decision Support, pp. 3-11, Berlin, Springer, 2018 